\title{
PANTS DECOMPOSITIONS OF RANDOM SURFACES
}

\author{
Larry Guth, Hugo Parlier and Robert Young
}

\begin{abstract}
Our goal is to show, in two different contexts, that "random" surfaces have large pants decompositions. First we show that there are hyperbolic surfaces of genus $g$ for which any pants decomposition requires curves of total length at least $g^{7 / 6-\varepsilon}$. Moreover, we prove that this bound holds for most metrics in the moduli space of hyperbolic metrics equipped with the Weil-Petersson volume form. We then consider surfaces obtained by randomly gluing euclidean triangles (with unit side length) together and show that these surfaces have the same property.
\end{abstract}

Any surface of genus $g, g \geq 2$, can be decomposed into three-holed spheres (colloquially, pairs of pants). We say that a surface has pants length $\leq l$ if it can be divided into pairs of pants by curves each of length $\leq l$. We say that a surface has total pants length $\leq L$ if it can be divided into pairs of pants by curves with the sum of the lengths $\leq L$. The pants length and total pants length measure the size and complexity of a surface. In particular, they describe how hard it is to divide the surface into simpler parts. One of the main open problems in this area is to understand how big the pants length of a genus $g$ hyperbolic surface can be. It would also be interesting to understand how big the total pants length of a genus $g$ hyperbolic surface can be. In this paper, we use a random construction to find hyperbolic surfaces with surprisingly large total pants length.

To put the paper in context, we review the known results about pants length and total pants length. In $[\mathrm{Be} 1,2]$, Bers proved that for each genus $g$, the supremal pants length of a genus $g$ hyperbolic surface is finite. This result is non-trivial because the moduli space of hyperbolic surfaces is not compact, and Bers' result gives information about the geometry of surfaces near the ends of moduli space. The supremal pants length of a genus $g$ hyperbolic surface is called the Bers constant, $\mathcal{B}_{g}$. Later work gave explicit estimates for the Bers constant. In [BuSe], Buser and Seppälä proved that every genus $g$ hyperbolic surface has pants length at most $C g$ (where $C$ is a constant independent of $g$ ). On the other hand, Buser [Bu1] gave examples of hyperbolic surfaces with pants length at least $c g^{1 / 2}$ for arbitrarily large $g$. Buser conjectured that the Bers constant of a hyperbolic surface is bounded by $C g^{1 / 2}$ [Bu2].

Keywords and phrases: Riemann surfaces, simple closed geodesics, Bers' constants, Teichmüller and moduli spaces

2010 Mathematics Subject Classification: Main: 32G15, Secondary: 53C20, 57M50, 60C05

Research of H.P. supported by Swiss National Science Foundation grant number PP00P2_128557. 
The total pants length has not been studied as much as the pants length, but it also seems like a natural invariant. Since a pants decomposition has $3 g-3$ curves in it, the estimate of Buser and Seppälä implies that every genus $g$ hyperbolic surface has total pants length at most $C g^{2}$. This is the best known general upper bound. In the other direction, it is easy to construct hyperbolic surfaces with total pants length at least $c g$ for every $g$ by taking covers of a genus 2 surface. The only previous nontrivial estimate comes from work of Buser and Sarnak on the geometry of certain arithmetic surfaces $[\mathrm{BuS}]$. They proved that there exist families of surfaces, one in each genus $g$, with the property that every topologically non-trivial curve has length at least $\sim \log g$. Since each curve in a pants decomposition is non-trivial, the total pants length of these arithmetic hyperbolic surfaces is at least $\sim g \log g$.

Now we have the background to state our main theorem.

Theorem 1. For any $\varepsilon>0$, a "random" hyperbolic surface of genus $g$ has total pants length at least $g^{7 / 6-\varepsilon}$ with probability tending to 1 as $g \rightarrow \infty$. In particular, for all sufficiently large $g$, there are hyperbolic surfaces with total pants length at least $g^{7 / 6-\varepsilon}$.

(To define a "random" hyperbolic surface we need a probability measure on the moduli space of hyperbolic metrics. We use the renormalized Weil-Petersson volume form. We discuss this notion of randomness more below.)

As another piece of context for our result, we mention the analogous questions for hyperbolic surfaces with small genus but many cusps. For simplicity, let's consider complete hyperbolic surfaces with genus 0 and $n$ cusps for $n \geq 3$. These surfaces also have pants decompositions, and pants length is defined in the same way. The arguments of Buser and Seppälä show that such a surface has pants length at most $C n$, and Buser's conjecture was that the pants length should be at most $C n^{1 / 2}$. Balacheff and Parlier proved this conjecture in $[\mathrm{BP}]$. In a more general context in $[\mathrm{BPS}]$, it is shown how to recuperate these results via Balacheff and Sabourau's diastolic inequality [BS]. There are also very good estimates for the total pants length in this context. Balacheff, Parlier and Sabourau showed that the total pants length of a hyperbolic surface with genus 0 and $n$ cusps is at $\operatorname{most} C n \log n$. It's easy to find examples where the total pants length is at least $c n$, so their bound is sharp up to logarithmic factors. The same authors went on to show that hyperelliptic genus $g$ hyperbolic surfaces have total pants length at most $\sim g \log g$. The hyperbolic surfaces in Theorem 1 are very different from hyperelliptic surfaces or from surfaces of genus 0 with many cusps.

Our lower bound is a lot stronger than the one coming from the Buser-Sarnak estimate. Instead of improving the trivial bound by a factor of $\log g$, we improve it by a polynomial factor $g^{1 / 6-\varepsilon}$. Let's take a moment to explain why it is difficult to prove such a lower bound. Almost all the random hyperbolic surfaces we construct have diameter around $\log g$. Therefore, they have lots of non-trivial curves with length around $\log g$. For example, we can make a basis for the first homology of the surface using curves of length around $\log g$. So there are lots of short curves that look like good candidates to include in a pants decomposition. The key issue seems 
to be that the curves in a pants decomposition need to be disjoint. After we pick a first curve, the second curve needs to avoid it. This cuts down our options for the second curve. Then the third curve needs to avoid the first two curves, so we have even fewer options. If the pants length of the surface is near $g^{7 / 6}$, then the curves "get in each other's way" so much that we have to pick dramatically longer curves to finish building a pants decomposition. This kind of effect looks difficult to prove because there are so many choices about how to choose the early curves. At the present time, for any particular hyperbolic surface, we cannot prove that the total pants length is any larger than $g \log g$. But for a random hyperbolic surface, the pants length is usually much larger than $g \log g$.

Our proof is essentially a counting argument. If a surface has total pants length at most $L$, we can construct it by gluing some hyperbolic pairs of pants with total boundary length $\leq L$. A hyperbolic pair of pants is determined by its boundary lengths, so the number (really, Weil-Petersson volume) of possible surfaces with total pants length $\leq L$ is governed by the number of possible ways of choosing these hyperbolic pairs of pants and gluing them together. We estimate this volume and show that if $L \leq g^{7 / 6-\varepsilon}$, then it is much smaller than the total volume of moduli space.

One difficulty with the above theorem is that it is not very constructive; there is no known algorithm to pick a random surface from the Weil-Petersson distribution on moduli space. Because of this, we will also prove a version of the theorem for combinatorial surfaces built by randomly gluing together equilateral triangles to form a closed surface. Suppose that we take $N$ Euclidean triangles of side length 1 , where $N$ is even. These triangles can be glued together to make a surface, and Gamburd and Makover [GM] showed that this surface usually has genus close to $(N+2) / 4$. We consider the set of triangulated surfaces obtained in this way. We identify surfaces that are simplicially isomorphic, and the equivalence classes form a kind of combinatorial "moduli space". We put the uniform measure on this finite set, so we can speak of a random combinatorial surface. (This definition goes back to Brooks and Makover [BrM], who studied the first eigenvalue of the Laplacian and the systole of random combinatorial surfaces.)

Theorem 2. For any $\varepsilon>0$, a random combinatorial surface with $N$ triangles has total pants length at least $N^{7 / 6-\varepsilon}$ with probability tending to 1 as $N \rightarrow \infty$.

The combinatorial metrics we study in Theorem 2 are not hyperbolic, so let us mention what is known about the pants lengths of arbitrary metrics on a surface of genus $g$. Here the natural question is how the pants length relates to the area. Generalizing his work with Seppälä, Buser proved that any (not necessarily hyperbolic) genus $g$ surface with area $A$ has pants length at most $C g^{1 / 2} A^{1 / 2}$ ([Bu2]). It's easy to give examples of surfaces with area $A$ and pants length at least $C A^{1 / 2}$. Generalizing his conjecture for hyperbolic metrics, Buser conjectured that any metric on a surface with area $A$ should have pants length at most $C A^{1 / 2}$. Recall that the systolic inequality for surfaces says that any surface of genus $g \geq 1$ and area $A$ has a non-trivial curve of length at most $C A^{1 / 2}$. If it's true, Buser's conjecture about 
pants lengths would greatly strengthen the systolic inequality. For an introduction to systolic geometry, see Chapter 4 of $[\mathrm{Gr}]$.

We include this alternate version of Theorem 1 for two reasons. Firstly, as mentioned above, the combinatorial case is more concrete than the hyperbolic case. It is easier to construct random combinatorial surfaces than random hyperbolic surfaces, and, although the proof of Theorem 2 is not as clean as the proof of Theorem 1, it is completely elementary and self-contained.

Secondly, the theorems and their proofs illustrate the parallels between the combinatorial case and the hyperbolic case. Comparing the work of Brooks-Makover and Makover-McGowan [MM] to recent work of Mirzakhani [Mi] suggests that for large $\mathrm{N}$, the counting measure on the combinatorial moduli space above may be close to the Weil-Petersson measure on moduli space. Our results support this. Indeed, the proofs of Theorems 1 and 2 follow similar lines and obtain similar estimates. For example, both proofs use an estimate of the total number (measure) of possible surfaces. A typical combinatorial surface with $N$ triangles has genus close to $N=g / 4$, and there are roughly $N^{N / 2} \approx g^{2 g}$ different surfaces with $N$ triangles. This is comparable to the volume of moduli space, which is also roughly $g^{2 g}$. Similar parallels appear between the number of possible pairs of pants with given boundary lengths and the number of possible ways to glue pairs of pants to get a surface.

There are many open questions related to the work in this paper. The central problem, understanding the maximal pants length of a genus $g$ hyperbolic surface, remains wide open. We found random surfaces to be useful in studying the total pants length, and there are also many questions about pants length for random surfaces. For instance, what is the average pants length of a random genus $g$ surface, and is the average pants length close to the Bers constant? Do the pants lengths of random genus $g$ surfaces concentrate near a single value? One also has the analogous questions for total pants length.

In several ways, arithmetic surfaces have properties similar to random surfaces. How does the pants length of an arithmetic surface compare with the pants length of a random surface? In particular, is it true that an arithmetic surface has pants length at least $g^{1 / 6-\varepsilon}$ ?

Many of the same questions arise for random combinatorial surfaces along with some new ones. For instance, the parallels between the hyperbolic and the combinatorial case suggest questions about the geometry of random combinatorial surfaces of different genuses. In the hyperbolic case, large total pants length seems to be a high-genus phenomenon: spheres with cusps have much smaller pants length than high-genus hyperbolic surfaces with the same area. Most random combinatorial surfaces have large genus, but we can also consider the set of combinatorial surfaces where the genus $g$ is fixed, but the number of triangles $N$ increases. One might hope that like the hyperbolic surfaces, these small-genus random surfaces might have simpler geometry than their high-genus counterparts.

The study of small-genus random surfaces is an active area in probability theory especially in recent years, and it has been an active area in physics for a long time. A lot of study has been paid to random metrics on $S^{2}$, and one of the key ideas is 
that there is essentially only one really natural random metric on $S^{2}$. Following this idea, one conjectures that different procedures for producing a random metric on $S^{2}$ should give the same result. In addition to the example above, where one glues $N$ triangles together and rejects any result which isn't a sphere, one can begin with the standard metric on $S^{2}$ and change it by a randomly chosen conformal factor (the conformal factor being a Gaussian free field) to get a "random planar map", an object studied in probability and physics - see [L]. Conjecturally, for large $N$, the probability distribution coming from gluing triangles converges to the probability distribution for the random conformal factor. For an introduction to these issues, see the survey article [L].

On the other hand, if we take $g$ large and $N$ to be roughly $4 g$, it seems that we get some kind of approximation to the moduli space of hyperbolic metrics. We can then ask questions about how closely such a random combinatorial surface resembles its random hyperbolic counterpart - what is its systole, first eigenvalue of Laplacian, or its Uryson width? What are its isoperimetric properties? How many balls of various radii are needed to cover it? What are its pants length and total pants length? And so on.

In the first section of the paper, we review the topology of pants decompositions. In the second section, we review some key background theorems about the WeilPetersson volume form, and we use them to prove Theorem 1. In the third section, we introduce the combinatorial moduli space and prove Theorem 2.

Notation. Many of the numbers we will be concerned with are super-exponential. For two numbers $A(x)$ and $B(x)$ that depend on a variable $x, A(x) \approx B(x)$ (resp. $A(x) \gtrsim B(x))$ will mean that they are equal (resp. the inequality holds) up to an exponential factor in $x$. For example, by Stirling's inequality, $g ! \approx g^{g}$.

Acknowledgement. We thank the referee for her or his helpful comments.

\section{Topological Types of Pants Decompositions}

Pants decompositions come in different topological types. Let us fix a surface $\Sigma$. A pants decomposition determines a trivalent graph where each pair of pants corresponds to a vertex and two vertices are joined by an edge if the corresponding pants share a boundary. (This trivalent graph may have multiple edges or loops.) We call this graph the topological type of the pants decomposition. We say that two pants decompositions are topologically equivalent if their topological types are isomorphic graphs. It's straightforward to check that if two pants decompositions are topologically equivalent, then there is a diffeomorphism of $\Sigma$ taking one to the other.

For example, if $\Sigma$ is a surface of genus 2, then it has two topological types of pants decomposition. The two types each correspond to trivalent graphs with two vertices. In the first case, there are three edges that go between the two vertices. In the second case, each vertex has one edge connecting it to the other vertex and one loop connecting it to itself. 
The first result that we need is an estimate for the number of different topological types of pants decomposition on a surface of genus $g$. In [Bo], Bollobas gave a precise asymptotic formula for the number of trivalent graphs. We need only the following cruder version of Bollobas's formula.

LEMma 1 (Bollobas). There are $\approx n^{n}$ trivalent graphs with $2 n$ vertices.

This lemma is cruder than Bollobas's result, and it also has a simpler proof. For reference, we include a proof here.

Proof. We start with $2 n$ labeled tripods and consider all the ways to glue them together to produce a labeled trivalent graph with labeled half-edges (or simply "labeled trivalent graph"). By a labeled tripod, we mean a vertex with three halfedges coming out of it, labeled 1, 2, and 3. Likewise, by a labeled trivalent graph, we mean a trivalent graph with labeled vertices where the three half-edges incident to each vertex have labels 1,2 , and 3 . The tripods have total degree $6 n$, and there are

$$
\frac{(6 n) !}{(3 n) ! 2^{3 n}} \approx n^{3 n}
$$

ways of dividing the $6 n$ half-edges into pairs, each of which corresponds to a labeled trivalent graph with vertices numbered $1, \ldots, 2 n$. An unlabeled trivalent graph occurs many times in this collection. The permutation group $S_{2 n}$ acts on the set of labeled graphs by permuting the vertex labels, and each orbit of the permutation group consists of isomorphic graphs. The number of equivalence classes is thus at most the number of orbits. If $G$ is a labeled graph, recall that its orbit has Card $S_{2 n} /$ Card $S_{2 n}^{G}$ elements, where $S_{2 n}^{G}$ is the stabilizer of $G$. The stabilizer of $G$ consists of permutations of the vertices which lead to an isomorphic graph. We can describe such a permutation in terms of the image of a basepoint in $G$ and a permutation of the neighbors of each vertex, so

$$
1 \leq \operatorname{Card} S_{2 n}^{G} \leq 2 n 6^{2 n} \lesssim 1
$$

Hence each orbit has $\approx n^{2 n}$ elements, and the number of orbits is $\approx n^{n}$.

Consequently, a pants decomposition of a genus $g$ surface has one of $\approx g^{g}$ possible topological types.

\section{The Moduli Space of Hyperbolic Metrics}

In this section we show that a random hyperbolic metric on a genus $g$ surface has total pants length at least roughly $g^{7 / 6}$ with very high probability. To begin, let us define what we mean by a random metric and make a precise statement.

We denote the moduli space of closed hyperbolic surfaces of genus $g$ by $\mathcal{M}_{g}$. The Weil-Petersson metric is a Riemannian metric on $\mathcal{M}_{g}$. We use the volume form of the Weil-Petersson metric to define volumes on moduli space. By renormalizing the Weil-Petersson volume form, we get a probability measure on moduli space. We take random metrics with respect to this probability measure. 
Theorem 1. For any $\varepsilon>0$, a random metric in $\mathcal{M}_{g}$ has total pants length at least $g^{\frac{7}{6}-\varepsilon}$ with high probability: the probability tends to 1 as $g \rightarrow \infty$.

Let us indicate the plan of our proof.

First, we observe that without loss of generality, we may assume that the curves in the pants decomposition are closed geodesics. Suppose we begin with a pants decomposition of a hyperbolic surface $\Sigma$. By definition, the pants decomposition consists of disjoint embedded curves $\gamma_{1}, \ldots, \gamma_{3 g-3}$ so that each component of the complement is a three-holed sphere. This is equivalent to just requiring that the curves $\gamma_{i}$ be disjoint and non-parallel: no two curves among them bound an annulus. Following a standard trick, we 'tighten' the curves $\gamma_{i}$ : we replace each curve $\gamma_{i}$ with a closed geodesic $\bar{\gamma}_{i}$ in its free homotopy class. By standard arguments in hyperbolic geometry, the closed geodesics will be embedded and disjoint, and no two of them will bound an annulus. Hence the curves $\bar{\gamma}_{i}$ give a pants decomposition of $\Sigma$ also, and they have smaller lengths than the curves $\gamma_{i}$.

From now on we assume that the curves in the pants decomposition are closed geodesics. So each three-holed sphere in the pants decomposition has a hyperbolic metric with geodesic boundary. We call such a pair of pants with such a metric a hyperbolic pair of pants. The classification of hyperbolic pairs of pants is easy to describe: the boundary curves may have any positive lengths, and for each choice of lengths there is a unique metric.

Now our plan consists of describing all the ways to glue together hyperbolic pairs of pants to make a closed hyperbolic surface, and then estimating the volume in moduli space covered by these surfaces.

To prove our theorem, we use two fundamental facts about the Weil-Petersson volume form. Recall that the Teichmüller space $\mathcal{T}_{g}$ denotes the space of hyperbolic metrics on a fixed surface of genus $g$, where two metrics are equivalent if they are related by an isometry isotopic to the identity. The moduli space $\mathcal{M}_{g}$ is the quotient of Teichmüller space $\mathcal{T}_{g}$ by the action of the mapping class group. The Weil-Petersson metric on Teichmüller space is a non-complete Kähler metric with negative sectional curvature, and is very much related to the hyperbolic geometry of surfaces. Although it is a very natural metric to consider, it is quite technical to define, so we refer the reader to $[\mathrm{W}]$ for details. The Weil-Petersson metric is invariant under the action of the mapping class group, so it descends to a metric on moduli space.

We will need the following result of Schumacher and Trapani [ST].

Background Theorem 1 (Asymptotic volume growth). The volume of moduli space $\mathcal{M}_{g}$ grows (up to an exponential factor) like $g^{2 g}$; i.e.

$$
\operatorname{Vol} \mathcal{M}_{g} \approx g^{2 g} \text {. }
$$

This result was an improvement of previous lower [P] and upper bounds [Gru].

The second background theorem expresses the Weil-Petersson volume form in a set of Fenchel-Nielsen coordinates. Before stating the result, we quickly recall Fenchel-Nielsen coordinates. 
Fix a pants decomposition of the genus- $g$ surface. We denote the curves in the pants decomposition by $\gamma_{1}, \ldots, \gamma_{3 g-3}$. Recall that $l_{i}$ and $\tau_{i}$, the length and twist parameters, define coordinates on the Teichmüller space $\mathcal{T}_{g}$. The length parameter $l_{i}$ measures the length of the shortest curve homotopic to $\gamma_{i}$ in the given metric; this is a positive real number. The twist parameter $\tau_{i}$ measures the twist in the gluing across this geodesic; this is a real number measured in units of length. Different length and twist parameters may correspond to the same point in $\mathcal{M}_{g}$; for instance, replacing $\tau_{i}$ by $\tau_{i} \pm l_{i}$ yields a metric isometric to the original one by a Dehn twist around $\gamma_{i}$.

The volume form for the Weil-Petersson metric has a very simple form in terms of these coordinates $[\mathrm{W}]$.

Background Theorem 2 (Wolpert). In Fenchel-Nielsen coordinates, the volume form of the Weil-Petersson metric is simply the standard volume form $d l_{1} \wedge \cdots \wedge$ $d l_{3 g-3} \wedge d \tau_{1} \wedge \cdots \wedge d \tau_{3 g-3}$.

The region in moduli space with total pants length less than $L$ in our fixed pants decomposition is covered by the following region of Teichmüller space:

$$
S=\left\{\left(l_{i}, \tau_{i}\right) \in T_{g} \mid \sum_{i} l_{i} \leq L, 0 \leq \tau_{i} \leq l_{i}\right\} .
$$

The Weil-Petersson volume of this set is the same as its volume in the standard Euclidean metric on $\mathbb{R}^{6 g-6}$ and it is not hard to estimate.

Lemma 2. If $1 \leq L \leq \exp (g)$, then $\operatorname{Vol} S \lesssim(L / g)^{6 g}$, where $\lesssim$ is taken with respect to $g$.

Proof. First consider the $(3 g-3)$-dimensional simplex defined by the inequalities $0<l_{i}, \sum_{i} l_{i} \leq L$. We denote this simplex by $\Delta_{L}$. By Fubini's theorem,

$$
\operatorname{Vol} S=\int_{\Delta_{L}} \prod_{i=1}^{3 g-3} l_{i} .
$$

By the arithmetic-geometric mean inequality,

$$
\prod l_{i} \leq\left(\frac{L}{3 g-3}\right)^{3 g-3} \lesssim\left(\frac{L}{g}\right)^{3 g} .
$$

Hence $\operatorname{Vol} S \lesssim \operatorname{Vol}\left(\Delta_{L}\right)(L / 3 g)^{3 g}$.

The volume of the simplex $\Delta_{L}$ may be calculated inductively using the formula for the volume of a pyramid. It is equal to $\frac{L^{3 g-3}}{(3 g-3) !} \lesssim(L / g)^{3 g}$.

REMARK. The calculation in this lemma is basically sharp: the region of Teichmüller space above has volume $\approx(L / g)^{6 g}$. The region of moduli space covered by this region of Teichmüller space has volume $\lesssim(L / g)^{6 g}$. (The volume in moduli space may be much smaller if the covering map is highly non-injective. We don't know how to estimate this effect.) 
Now let $\mathcal{M}_{g}(\leq L) \subset \mathcal{M}_{g}$ denote the subset of hyperbolic metrics that admit pants decompositions of total length $\leq L$.

If $E$ denotes a topological type of pants decomposition, we let $\mathcal{M}_{g}(\leq L, E) \subset$ $\mathcal{M}_{g}(\leq L)$ denote the the subset of hyperbolic metrics that admit a pants decomposition of type $E$ and total length $\leq L$. For each $E$, the calculation in Fenchel-Nielsen coordinates shows that the volume of $\mathcal{M}_{g}(\leq L, E)$ is $\lesssim(L / g)^{6 g}$. There are $\approx g^{g}$ different topological types $E$. Every pants decomposition belongs to one of these $\approx g^{g}$ types, and so the volume of $\mathcal{M}_{g}(\leq L)$ is $\lesssim L^{6 g} g^{-5 g}$.

As we saw above, the volume of $\mathcal{M}_{g}$ is $\approx g^{2 g}$. If we set $L=g^{(7 / 6)-\varepsilon}$, then we see that the volume of $\mathcal{M}_{g}(\leq L) \lesssim g^{(2-6 \varepsilon) g}$. Recalling the definition of $\lesssim$ and $\gtrsim$, we see that the volume of $\mathcal{M}_{g}$ is at least $c e^{-c g} g^{2 g}$, while the volume of $\mathcal{M}_{g}\left(\leq g^{(7 / 6)-\varepsilon}\right)$ is at most $C e^{C g} g^{(2-6 \varepsilon) g}$. So for $g$ sufficiently large, the volume of $\mathcal{M}_{g}$ is much larger than the volume of $\mathcal{M}_{g}\left(\leq g^{(7 / 6)-\varepsilon}\right)$. This proves Theorem 1 .

\section{The Combinatorial Viewpoint}

If $N$ is even, one can construct a oriented surface by gluing together $N$ triangles (we allow edges of the same triangle to be glued together and allow edges to form loops). We call the corresponding CW-complex a combinatorial surface. We declare two combinatorial surfaces to be equivalent if there is a homeomorphism which sends edges to edges and faces to faces, and we define $\operatorname{Comb}_{N}$ to be the set of equivalence classes of such surfaces with $N$ triangles. Gamburd and Makover [GM] showed that as $N \rightarrow \infty$, a random element of $\operatorname{Comb}_{N}$ has genus at least $(1 / 4-\varepsilon) N$ with high probability (for a precise estimate, see $[\mathrm{G}]$ ), so $\mathrm{Comb}_{N}$ is a rough combinatorial equivalent of $\mathcal{M}_{N / 4}$.

We think of each triangle in a combinatorial surface as a Euclidean equilateral triangle with side length 1 . In this way, each combinatorial surface in $\operatorname{Comb}_{N}$ has a metric on it. In particular, we can define its pants length and total pants length. We also have a good notion of a random combinatorial surface given by the counting measure on $\operatorname{Comb}_{N}$. Using these definitions, we get the following combinatorial version of our main result.

Theorem 2. For any $\varepsilon>0$, a random combinatorial surface in $\mathrm{Comb}_{N}$ has total pants length at least $N^{7 / 6-\varepsilon}$ with probability tending to 1 as $N \rightarrow \infty$.

The proof of Theorem 2 is morally analogous to the proof of Theorem 1 . It is more elementary, because it does not rely on the Weil-Petersson metric, but there are also some additional subtleties.

One of the key observations in the proof of Theorem 1 was that a hyperbolic surface of total pants length $L$ can be cut into hyperbolic pairs of pants whose boundaries have total length at most $2 L$. The main subtlety in the proof of Theorem 2 is to find the right combinatorial analogue for this step.

Suppose we start with an arbitrary pants decomposition of a combinatorial surface $\Sigma$. The curves $\gamma_{i}$ are arbitrary curves, and so the pairs of pants in the decomposition do not have combinatorial structures. To get a combinatorial decomposition, 
we need the $\gamma_{i}$ to be combinatorial curves. For each $i$, we can approximate $\gamma_{i}$ by a combinatorial curve $\bar{\gamma}_{i}$ which is homotopic to $\gamma_{i}$ and has comparable length. At this point, some problems appear: the curves $\bar{\gamma}_{i}$ need not be embedded and need not be disjoint. If the $\bar{\gamma}_{i}$ are chosen carefully, however, we can still express $\Sigma$ as the union of combinatorial pairs of pants, glued along the $\bar{\gamma}_{i}$. A combinatorial pair of pants will consist of triangles and edges, but some of the edges may not border any of the triangles. These isolated edges can be thought of as 'infinitely thin' pieces of surface. We will show that we can choose the $\bar{\gamma}_{i}$ to be combinatorial geodesics, so that each one has minimal length compared to all combinatorial curves in its homotopy class.

At this point, there is a further problem. A hyperbolic pair of pants is determined by the lengths of its boundary curves. But there are many different combinatorial pairs of pants with the same boundary lengths. When we count how many ways we can glue together combinatorial pairs of pants with given boundary lengths, we get a large unwanted factor coming from the different choices for a pair of pants with fixed boundary curves. The underlying cause of this problem is that combinatorial geodesics - unlike hyperbolic ones - are not unique. The solution to this problem is to consider only special combinatorial pants decompositions which we call 'tight pants decompositions'. We define these below. Roughly speaking, they are pants decompositions of minimal complexity in an appropriate sense.

With this well-chosen definition, the analogy runs smoothly. In section 3.1, we prove that $\operatorname{Comb}_{N}$ has cardinality $\approx N^{N / 2}$, but that for any fixed $g$, the number of $N$-triangle combinatorial surfaces of genus $g$ grows only exponentially. In section 3.2, we introduce combinatorial pants decompositions and tight combinatorial pants decompositions. We show that any pants decomposition can be improved to make a tight combinatorial pants decomposition. In section 3.3, we count the number of tight combinatorial pants decompositions of total length $\leq L$.

3.1 Counting Combinatorial Surfaces. The goal of this part is to count surfaces that lie in our combinatorial moduli space. Our main goal is to prove that the cardinality of $\mathrm{Comb}_{N}$ is $\approx N^{N / 2}$. Since most surfaces in $\mathrm{Comb}_{N}$ have genus close to $N / 4$, this is analogous to the fact that the Weil-Petersson volume of moduli space of surfaces of genus $g$ is $\approx g^{2 g}$.

Over the course of our argument, we will need to consider the set of combinatorial surfaces with a particular genus. Let $\mathrm{Comb}_{N} \operatorname{Mod}_{g, k}$ denote all combinatorial surfaces of genus $g$ with $k$ boundary components made from $N$ triangles. Again, we will allow two edges of the same triangle to be glued together, and again we consider surfaces up to homeomorphisms preserving edges and faces.

For fixed $g$ and $k$, we will see that the cardinality of $\operatorname{Comb}_{N} \operatorname{Mod}_{g, k}$ grows only exponentially with $N$.

We begin by studying the cardinality of $\operatorname{Comb}_{N}$.

LEMMA 3 (Combinatorial volume growth).

$$
\operatorname{Card}\left(\operatorname{Comb}_{N}\right) \approx N^{N / 2} \text {. }
$$


Proof. If $\Sigma \in \mathrm{Comb}_{N}$, we can construct a trivalent graph with $N$ vertices by letting the vertices of the graph be the faces of $\Sigma$ and connecting vertices whose corresponding faces share an edge. Given a trivalent graph, we can construct a surface with the corresponding pattern of gluings, so by Lemma $1, \operatorname{Card}\left(\operatorname{Comb}_{N}\right) \gtrsim N^{N / 2}$.

On the other hand, many surfaces might correspond to the same graph, since the graphs do not record which of the three edges of each triangle is glued to which other edge. With this information, a graph uniquely identifies a surface, but there are only $6^{N}$ ways to add this information to the graph, so $\operatorname{Card}\left(\operatorname{Comb}_{N}\right) \lesssim N^{N / 2}$ as well.

In contrast, the number of ways to triangulate a surface of a fixed genus with many triangles grows only exponentially:

LEMmA 4. For any $g \geq 0, k \geq 0$,

$$
\operatorname{Card}\left(\operatorname{Comb}_{n} \operatorname{Mod}_{g, k}\right) \lesssim e^{n} .
$$

In other words,

$$
\operatorname{Card}\left(\operatorname{Comb}_{n} \operatorname{Mod}_{g, k}\right) \leq C(g, k) e^{n} .
$$

Proof. In the special case that $(g, k)=(0,1)$ (i.e. for triangulations of a disk), this follows from a result of Brown [Bro]; we will reduce the general case to the case of a disk.

Brown counted the number of rooted simplicial triangulations of the disk, that is, triangulations with a marked oriented boundary edge such that the endpoints of each edge are distinct and no face is glued to itself, and showed that the number of such triangulations with $j+3$ boundary vertices and $k$ interior vertices is

$$
\frac{2(2 j+3) !(4 k+2 j+1) !}{(j+2) ! j ! k !(3 k+2 j+3) !} .
$$

Restating this in terms of the number $n=j+2 k+1$ of triangles, we find that the number of triangulations is

$$
\frac{2(2 j+3) !(2 n-1) !}{(j+2) ! j !\left(\frac{n-j-1}{2}\right) !\left(\frac{3 n+j-3}{2}\right) !}
$$

where we require that $0 \leq j \leq n-1$ and $j \equiv n-1 \bmod 2$. Call this number $\theta(n, j)$. Our combinatorial surfaces differ from Brown's in that the two endpoints of an edge may be identified, but any element of $\mathrm{Comb}_{n} \operatorname{Mod}_{0,1}$ can be barycentrically subdivided twice to get a simplicial triangulation. We thus find that

$$
\operatorname{Card}\left(\operatorname{Comb}_{n} \operatorname{Mod}_{0,1}\right) \leq \sum_{\substack{j=0 \\ j \equiv 6 n-1(\bmod 2)}}^{6 n-1} \theta(6 n, j) .
$$

Since $j \leq n-1$, there is a $c$ such that

$$
\theta(n, j) \leq e^{c n} \frac{(2 n-1) !}{\left(\frac{n-j-1}{2}\right) !\left(\frac{3 n+j-3}{2}\right) !}=e^{c n}(2 n-1)\left(\begin{array}{c}
2 n-2 \\
\frac{n-j-1}{2}
\end{array}\right) \leq e^{c n} 2^{2 n-2}(2 n-1)
$$


so there is a $c^{\prime}$ such that for all $n>0$,

$$
\sum_{j=0}^{n-1} \theta(6 n, j) \leq e^{c^{\prime} n} .
$$

Now consider $\operatorname{Comb}_{N} \operatorname{Mod}_{g, k}$. If $\Sigma$ is a triangulated genus- $g$ surface with $k$ holes and $(g, k) \neq(0,0)$, we can cut it along non-separating simple closed curves or simple arcs between boundary components until we get a disk; this takes at most $3 g+k-1$ cuts. We can thus obtain any element of $\operatorname{Comb}_{N} \operatorname{Mod}_{g, k}$ by performing at most $3 g+k-1$ gluings on an element of $\mathrm{Comb}_{n} \operatorname{Mod}_{0,1}$. Each gluing identifies two edge paths on the boundary, so a gluing is determined by the endpoints of the paths that are glued. There are at most $(3 n)^{4}$ ways to perform each gluing, so as long as $(g, k) \neq(0,0)$,

$$
\operatorname{Card}\left(\operatorname{Comb}_{n} \operatorname{Mod}_{g, k}\right) \leq e^{c^{\prime} n}(3 n)^{4(3 g+k-1)} \lesssim e^{n} .
$$

For the case that $(g, k)=(0,0)$, note that if $\Sigma$ is a triangulation of a sphere, then we can obtain a triangulation of the disc by cutting along an edge of $\Sigma$, so

$$
\operatorname{Card}\left(\operatorname{Comb}_{n} \operatorname{Mod}_{0,0}\right) \leq e^{c^{\prime} n} \lesssim e^{n}
$$

as desired.

3.2 Combinatorial pants decompositions. In this part, we define pants decompositions of combinatorial surfaces and their lengths. We then focus our interest on pants decompositions of minimal length, and we show that in the isotopy class of such a pants decomposition there is always a pants decomposition of a particular type, called a tight pants decomposition.

A pants decomposition of a surface of genus $g$ is a maximal set of disjoint and freely homotopically distinct non-trivial simple closed curves. A pants decomposition always contains $3 g-3$ curves, and its complementary region consists of a set of $2 g-2$ three holed spheres, or pairs of pants. To define a pants decomposition in the combinatorial setting, we focus on these pairs of pants.

A combinatorial pair of pants will consist of a simplicial complex equipped with some boundary curves. Let $\Delta$ be a simplicial complex which is a deformation retract of a three-holed sphere $M_{0,3}$. If we consider $\Delta$ as a subset of $M_{0,3}$, this implies that a regular neighborhood of $\Delta$ is a three-holed sphere. The boundary curves of this three-holed sphere correspond to simplicial curves in the boundary of $\Delta$, and when $\Delta$ is equipped with these boundary curves, we call it a combinatorial pair of pants. These curves inherit an orientation from $M_{0,3}$. Note that $\Delta$ need not be a manifold; for instance, it could be two vertices connected by three edges. In general, $\Delta$ may contain edges that are not boundaries of triangles. We call such edges stranded edges.

We can glue pairs of pants to get surfaces. If $P_{1}, \ldots, P_{2 g}$ are combinatorial pairs of pants, each one has three boundary components, and we can identify pairs of boundary components which have the same length. Like its geometric analogue, 
there are many ways to identify the same pair of boundary components, and these ways differ by a shift; by fixing a basepoint on each boundary curve, we can define twist parameters for the gluing. If we glue all of the boundary curves in pairs, we obtain a complex $\bigcup P_{i} / \sim$; this may not be a surface, because there may still be edges which are not part of a triangle. If $\bigcup P_{i} / \sim$ is isomorphic to a triangulated surface $\Sigma$, we call the collection of the $P_{i}$, the gluing instructions, and the isomorphism a combinatorial pants decomposition of $\Sigma$. The boundary curves of the $P_{i}$ project to simplicial curves on $\Sigma$; we call these the boundary curves of the pants decomposition. If the boundary curves have minimal (combinatorial) length in their free homotopy classes, we say that the pants decomposition is minimal. We give $\Sigma$ a metric so that each triangle is isometric to an equilateral triangle with side length 1 .

We will show that a geometric pants decomposition gives rise to a minimal combinatorial pants decomposition. Recall that a Lipschitz closed curve on a triangulated surface is homotopic to a simplicial curve whose length is bounded by a constant times the length of the original curve:

Lemma 5. Let $\alpha: S^{1} \rightarrow \Sigma$ be a Lipschitz curve on a triangulated surface $\Sigma$. There is a simplicial curve $\lambda$ on $\Sigma$ which is homotopic to $\alpha$ and whose length satisfies

$$
\ell(\lambda) \leq 2 \ell(\alpha) .
$$

Proof. For any $\varepsilon>0$, we can perturb $\alpha$ to get a smooth curve $\alpha^{\prime}$ of length $\ell\left(\alpha^{\prime}\right) \leq$ $\ell(\alpha)+\varepsilon$ which avoids vertices of $\Sigma$ and intersects its edges transversely. The edges of the triangulation cut $\alpha^{\prime}$ into finitely many arcs, each contained in a single triangle. Each arc $a$ cuts the boundary of its triangle into two arcs. We homotope the arc $a$ to the shortest of these two boundary arcs (say $b$ ). By elementary euclidean geometry, we have $\ell(b) \leq 2 \ell(a)$. As such, the resulting curve $\beta$ satisfies $\ell(\beta) \leq 2 \ell(\alpha)$.

The resulting curve is not necessarily a simplicial curve as it may go partway along an edge and then backtrack. A further homotopy removes this backtracking and decreases the length. The resulting curve $\lambda$ is now a simplicial curve and has length at most the length of $\beta$. This proves the lemma.

We can now focus our attention on the equivalent statement for full pants decompositions.

Proposition 1. Let $\Sigma$ be a triangulated surface and let $\alpha_{1}, \ldots, \alpha_{k}: S^{1} \rightarrow \Sigma$ be the boundary curves of a pants decomposition for $\Sigma$. There is a minimal combinatorial pants decomposition of $\Sigma$ with boundary curves $\lambda_{1}, \ldots, \lambda_{k}: S^{1} \rightarrow \Sigma$ such that for all $i, \lambda_{i}$ is homotopic to $\alpha_{i}$ and $\ell\left(\lambda_{i}\right) \leq 2 \ell\left(\alpha_{i}\right)$.

Proof. The first step is to approximate the $\alpha_{i}$ by simplicial curves. For all $i$, let $\beta_{i}$ be a simplicial closed curve of minimal length which is homotopic to $\alpha_{i}$. In general, the $\beta_{i}$ may share edges; we will subdivide $\Sigma$ and make them disjoint.

We first duplicate the edges of $\Sigma$ so that the $\beta_{i}$ do not share edges. If $e=(x, y)$ is an edge of $\Sigma$ which occurs $n \geq 2$ times in the $\beta_{i}$, we replace $e$ with $n-1$ bigons glued edge-to-edge. We make no changes to edges which do not occur or occur only once. Now we replace the vertices of $\Sigma$; if a vertex has degree $d$, we replace it with a $d$-gon 
which we call a vertex polygon. Each incoming edge connects to one vertex of this $d$-gon; this makes the bigons created in the previous step into rectangles which we call edge rectangles. Each edge rectangle has two edges which are shared by vertex polygons and two edges which connect vertex polygons; we call the edges which connect vertex polygons long edges. Call the resulting complex $\Sigma^{\prime}$. This complex is homeomorphic to $\Sigma$, and there is a natural map $p: \Sigma^{\prime} \rightarrow \Sigma$ which collapses the edge rectangles to edges and the vertex polygons to vertices; this map sends long edges to edges of $\Sigma$ homeomorphically.

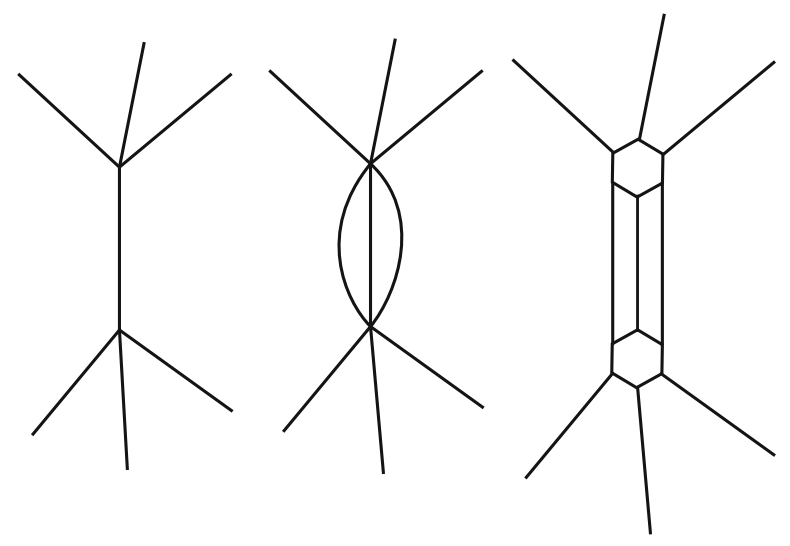

Figure 1: From $\Sigma$ to $\Sigma^{\prime}$

We call a curve semi-simplicial if it consists of alternating long edges and curves in vertex polygons; the image of a semi-simplicial curve under $p$ is thus a simplicial curve. If $\gamma$ is a semi-simplicial curve, we define its length by $\ell(\gamma):=\ell(p(\gamma))$. We can lift each of the $\beta_{i}$ to semi-simplicial curves in $\Sigma^{\prime}$ by replacing edges of $\beta_{i}$ with corresponding long edges and replacing vertices of $\beta_{i}$ with curves in the vertex polygons. There are enough long edges in $\Sigma^{\prime}$ that we can ensure that no long edge is used twice, so this gives us curves $\beta_{i}^{\prime}$ in $\Sigma^{\prime}$ which intersect only in the vertex polygons. We may assume that at most two curves intersect at a point and that all intersections are transverse.

A standard argument allows us to perform surgeries to make these curves disjoint. If two curves $\beta_{i}^{\prime}$ and $\beta_{j}^{\prime}$ intersect, then the fact that the two curves are homotopic to disjoint curves implies that there is a bigon between $\beta_{i}^{\prime}$ and $\beta_{j}^{\prime}$. That is, there is a subsegment $\gamma_{1}$ of $\beta_{i}^{\prime}$ and a segment $\gamma_{2}$ of $\beta_{j}^{\prime}$ which have the same endpoints and are homotopic relative to their endpoints. We call these endpoints $x$ and $y$. Because $\beta_{i}^{\prime}$ and $\beta_{j}^{\prime}$ have minimal length, we have $\ell\left(\gamma_{1}\right)=\ell\left(\gamma_{2}\right)$. We can eliminate the bigon by swapping $\gamma_{1}$ and $\gamma_{2}$ and deforming the resulting curves near $x$ and $y$ to eliminate the two intersection points. Likewise, if a curve $\beta_{i}^{\prime}$ intersects itself, then the fact that the curve is homotopic to a simple curve implies that there is a bigon bounded by two segments of $\beta_{i}^{\prime}$ and we can eliminate this bigon in the same way. In either case, this operation reduces the number of intersection points by two, so we can repeat it to eliminate all intersection points. Since we have only swapped subsegments of 
curves and performed homotopies inside vertex polygons, the resulting curves are still semi-simplicial and still have minimal length; call them $\beta_{1}^{\prime \prime}, \ldots, \beta_{k}^{\prime \prime}$.

We will get a combinatorial pants decomposition of $\Sigma$ by cutting $\Sigma^{\prime}$ along these curves and collapsing edge rectangles and vertex polygons. The curves $\beta_{1}^{\prime \prime}, \ldots, \beta_{k}^{\prime \prime}$ are the boundary curves of a geometric pants decomposition of $\Sigma^{\prime}$ into subsurfaces $P_{1}, \ldots, P_{2 k / 3}$. Each of these subsurfaces is homeomorphic to a pair of pants (i.e. a three-holed sphere), and is the union of edge rectangles, subsets of the vertex polygons, and faces of $\Sigma^{\prime}$ which come from triangles of $\Sigma$. If we collapse each edge rectangle in $P_{i}$ to an edge and each connected component of a vertex polygon to a vertex, we obtain a complex $P_{i}^{\prime}$ which comes with a map $p_{i}: P_{i}^{\prime} \rightarrow \Sigma$. The boundary curves of $P_{i}$ correspond to simplicial curves in $P_{i}^{\prime}$, and with these boundary curves, $P_{i}^{\prime}$ forms a combinatorial pair of pants. Furthermore, gluing the $P_{i}^{\prime}$ along these boundary curves reconstructs the original surface $\Sigma$, making them a combinatorial pants decomposition of $\Sigma$. The boundary curves of this pants decomposition are $\lambda_{i}:=p\left(\beta_{i}^{\prime \prime}\right)$; as required, $\lambda_{i}$ is homotopic to $\alpha_{i}$, and since it has minimal combinatorial length, its length is no more than a constant factor larger than that of $\alpha_{i}$.

Let $\Sigma$ be a genus $g$ surface and let $P_{1}, \ldots, P_{2 g-2}$ be the pants in a combinatorial pants decomposition of $\Sigma$. We can view a combinatorial pair of pants $P$ as a collection of clusters connected by strands; indeed, we will construct the cluster graph $G$ of $P$, a graph whose vertices correspond to clusters of $P$ and whose edges correspond to strands. We construct this graph as follows (see Figure 2):

1. For all vertices $v \in P$, if the link of $v$ has $d$ connected components and more than one is an interval, replace $v$ by a star with $d$ edges.

2. Shrink paths of edges to single edges.

3. Shrink groups of triangles which are glued along edges to single vertices.

Each vertex of $G$ corresponds to a group of triangles (indeed, a submanifold of $P$ with boundary) or a single point; we call these clusters. We will call a single-point cluster degenerate. Each edge corresponds to a path of stranded edges of $P$ (possibly a path of length zero); we call these strands. Since each cluster corresponds to a vertex of $G$, we can define the degree of a cluster to be the degree of the corresponding vertex.

The interior of a cluster can be homeomorphic to a disk, a cylinder or a three holed sphere, and we call it disk-type, cylinder-type, or three-holed-sphere-type accordingly. If a cluster is a single point, we say it is disk-type. If the pants decomposition was minimal, then a disk-type cluster can have degree two, three, or four. A cylinder-type cluster has degree one or two, and a three-holed-sphere-type cluster has degree zero. A pair of pants $P$ is called tight if none of its disk-type clusters have degree 2. Such a cluster will be called a loose disk. A minimal length pants decomposition is called tight if all of its pants are tight, i.e. do not contain any loose disks.

We are now able to introduce the main result of this section.

Lemma 6. Any combinatorial surface $\Sigma$ admits a minimal length tight pants decomposition. 


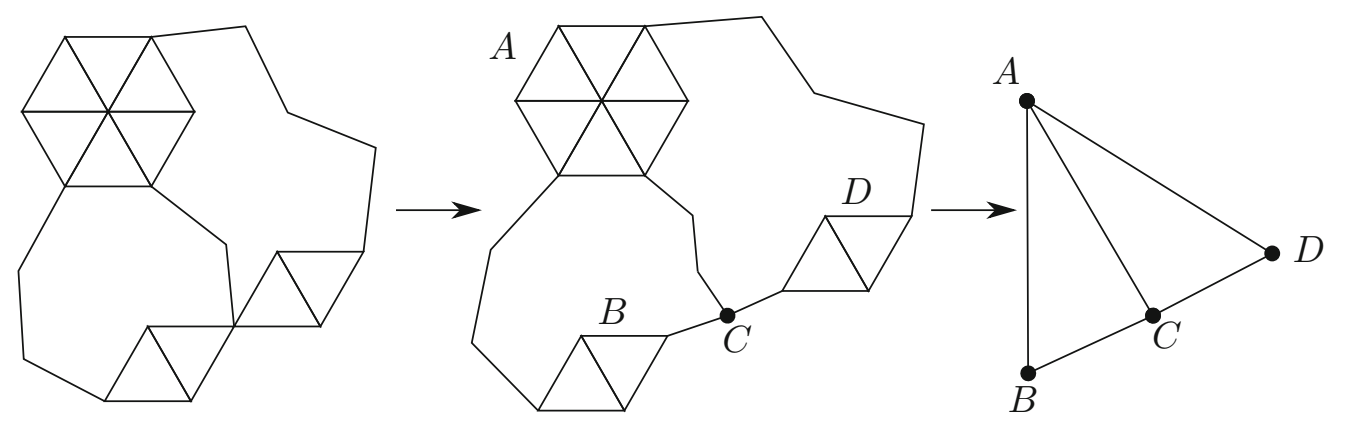

Figure 2: The cluster graph. This pair of pants has four disk-type clusters, one of which $(C)$ is degenerate and two of which $(B, D)$ are loose disks.

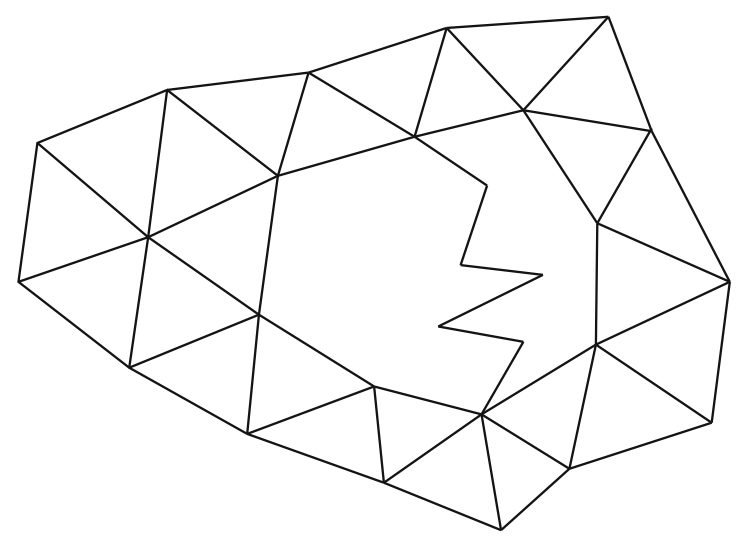

Figure 3: A pair of pants with a annulus-type cluster

Proof. We need to show that if a minimal pants decomposition has pants with loose disks we can isotope the curves to remove the disk from the pair of pants without increasing length. We further need to make sure that by doing so we are not just moving a loose disk somewhere else, and that in fact we will have reduced the number of loose disks.

The key technique in this proof is sliding a loose disk from one pair of pants to another. Let $D$ be a loose disk which is part of a pair of pants $P$ in a minimal pants decomposition, and say that $P$ has boundary curves $\gamma_{a}: S_{a} \rightarrow P, a=1,2,3$. Since $D$ is a loose disk, there are two strands which enter $D$, say at vertices $x_{1}$ and $x_{2}$. These two vertices divide the boundary of $D$ into two paths, and since the boundary curves of $P$ have minimal length, the two paths have equal length. We denote them $c, c^{\prime}:[0, \ell] \rightarrow P$, and we can choose $n$ and $n^{\prime}$ so that $c$ is a subsegment of $\gamma_{n}$ and $c^{\prime}$ is a subsegment of $\gamma_{n^{\prime}}$. If $P$ is glued to $Q$ along $\gamma_{n}$, then we can slide that common boundary curve over $D$ to transfer $D$ from $P$ to $Q$. This has the effect of cutting $D$ out of $P$ (leaving $c^{\prime}$ ) and gluing it on to $Q$ along $c$, and it produces a new pants 

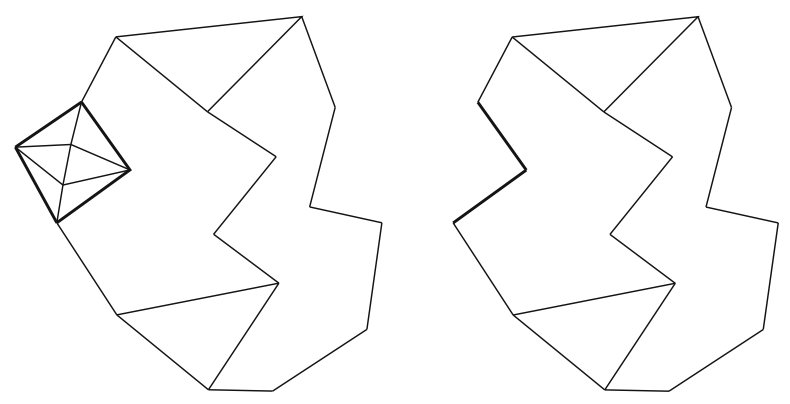

Figure 4: Removing a loose disk to get a tight pair of pants

decomposition of $\Sigma$. We call this process sliding $c$ to $c^{\prime}$. Furthermore, since $c$ and $c^{\prime}$ must have equal lengths, the lengths of the boundary curves are unchanged and the new pants decomposition is also minimal.

After such a slide, the pants decomposition differs from the original only around $D$. All the clusters of the original decomposition have counterparts in the new one, except possibly for clusters in $P$ and $Q$; the move deleted one cluster from $P$ and added at most one to $Q$, depending on whether $D$ was glued to a cluster or to a strand. If $D$ was glued to a cluster, then the move reduced the number of loose disks by one. Otherwise, the slide glues $D$ to a strand of $Q$. In this case, after the slide $D$ becomes a loose disc in $Q$ bounded by segments $c_{1}$ and $c_{1}^{\prime}$.

We will inductively define a sequence $X_{0}, X_{1}, \ldots, X_{k}$ of pants decompositions which all differ by slides. Each $X_{i}$ except $X_{k}$ will have a loose disk $D_{i}$ in a pair of pants $P_{i}$. This disk will be isomorphic to $D$ and which is bounded by two curves $c_{i}:[0, \ell] \rightarrow P_{i}$ and $c_{i}^{\prime}:[0, \ell] \rightarrow P_{i}$. Recall that $\Sigma$ is a quotient of the pairs of pants in $X_{i}$; let $\mu_{i}: P_{i} \rightarrow \Sigma$ be the restriction of the quotient map to $P_{i}$. We will require that $\mu_{i} \circ c_{i}:[0, \ell] \rightarrow \Sigma$ be the same curve for all $i<k$ and likewise for $\mu_{i} \circ c_{i}^{\prime}:[0, \ell] \rightarrow \Sigma$, and we will define $f:=\mu_{i} \circ c_{i}$ and $f^{\prime}:=\mu_{i} \circ c_{i}^{\prime}$.

Let $X_{0}$ be the original decomposition of $\Sigma$; this has a loose disk $D_{0} \cong D$ bounded by $c_{0}:=c$ and $c_{0}^{\prime}:=c^{\prime}$. We construct $X_{i+1}$ from $X_{i}$ by sliding $c_{i}$ to $c_{i}^{\prime}$. If this reduces the number of loose disks, we stop, letting $k=i+1$; otherwise, $D_{i}$ corresponds to a loose disk of $X_{i+1}$, bounded by $c_{i+1}$ and $c_{i+1}^{\prime}$, and we continue. We claim that this process eventually stops.

By way of contradiction, say that the process does not stop. Sliding $c_{i}$ to $c_{i}^{\prime}$ affects the boundary curves of $X_{i}$ by replacing an occurrence of $f$ by $f^{\prime}$. If the process does not stop, then we can replace $f$ by $f^{\prime}$ infinitely many times. In particular, each edge of $\Sigma$ occurs as many times in $f$ as it does in $f^{\prime}$, so each edge of $\Sigma$ occurs an even number of times in $\mu(\partial D)$ (indeed, either 0 or 2). Consequently, $\mu(D)=\Sigma$. Since $\mu$ is injective on the interior of $D$, this means that we can obtain $\Sigma$ by gluing the edges of $D$ together.

If $w=c(0)$ or $w=c(\ell)$, we call $w$ an endpoint of $D_{0}$. We claim that for all $v \in \Sigma, \mu^{-1}(v)$ contains at most 2 non-endpoint vertices of $D$. Say $v$ is such that $\left\{w_{1}, w_{2}, w_{3}\right\} \subset \mu^{-1}(v)$ for some 3 distinct non-endpoint vertices $w_{1}, w_{2}, w_{3} \in D$. The 
link of $v$ is a circle, and it contains 3 intervals corresponding to the links of the $w_{i}$. Let $S \subset \operatorname{link} v$ be the complement of the interiors of these intervals; this consists of three connected components, $S_{1}, S_{2}$, and $S_{3}$. If a length-2 segment of a boundary curve of $X$ passes through $v$, there are $j$ and $k$ such that it approaches $v$ from the direction of $S_{j}$ and leaves $v$ in the direction of $S_{k}$. We call this a $j k$-segment. Note that the only $j k$-segments of $\partial D$ with $j \neq k$ are those centered at the $w_{i}$. Since $D$ and the $S_{a}$ 's are unaffected by repeatedly sliding $c$ to $c^{\prime}$, we can discuss $j k$-segments of the boundary curves of the $X_{i}$ too.

One of the $w_{i}$ is in the image of $c$; say $w_{1}=c(x)$, so that $f$ passes through $v$ at $x$, and number the $S_{i}$ so that this is a 12-segment. Replacing $f$ by $f^{\prime}$ deletes this segment, and we claim that replacing $f$ by $f^{\prime}$ decreases the number of 12 -segments by one. The path $f^{\prime}$ has no 12 -segments, so it only remains to check that replacing $f$ by $f^{\prime}$ can't introduce new 12-segments centered at the endpoints of $D$. But if $f(0)=f^{\prime}(0)=v$ or $f(\ell)=f^{\prime}(\ell)=v$, then $f$ and $f^{\prime}$ both leave $v$ in the direction of the same $S_{i}$, so a $j k$-segment centered at an endpoint remains a $j k$-segment when $f$ is replaced by $f^{\prime}$. Since the number of 12 -segments in $X$ is finite, the process terminates after a finite number of slides.

Thus, $\Sigma$ can be obtained by gluing a disc to itself along its edges; the resulting gluing has one face, namely $D, \ell$ edges, and at least $(\ell-1)$ vertices. Thus, if the process does not terminate, then $\Sigma$ has genus at most 1 , which is a contradiction.

3.3 Counting tight pants decompositions. The goal of this part is to count the number of tight pants decompositions of bounded length.

Main Estimate. There is a $c>0$ such that the number of triangulated surfaces in Comb $_{N}$ with genus $g$ and (tight) pants decompositions of total length at most $L$ is $\leq \exp (c N) g^{g}(L / g)^{6 g}$.

First we will count the number of different tight pairs of pants with boundary curves of controlled length. Next we will count the number of ways of gluing these pants together into a surface.

LEMma 7. There is a $c_{0}>0$ such that the number of tight pairs of pants with boundary curves of lengths $l_{1}, l_{2}$, and $l_{3}$ and with $A$ triangles is $\leq c_{0} e^{c_{0} A}$.

Proof. A tight pair of pants consists of some clusters (which may be collections of triangles or may be single points) joined by some strands. In the last subsection, we constructed the cluster graph of a combinatorial pair of pants, a graph whose vertices correspond to clusters of triangles (possibly degenerate) and whose edges correspond to strands. We call the collection of the cluster graph of a pair of pants and the type of each cluster its combinatorial type. There are five possible combinatorial types for a tight pair of pants:

1. One disk-type cluster, joined to itself by two strands;

2. One annulus-type cluster, joined to itself with one strand;

3. One three-holed-sphere-type cluster and no strands;

4. Two disk-type clusters, joined by three strands running between them; 
5. Two disk-type clusters and three strands, one connecting the clusters, and one joining each cluster to itself.

One can show that these are all of the possible types by an Euler characteristic argument. Each cluster of triangles has to be a subsurface with genus 0 and at most 3 boundary components, so there are finitely many types of cluster. If $c$ is the number of clusters, $s$ is the number of strands, and $b$ is the total of the first Betti numbers of the clusters in a pair of pants $P$, then the first Betti number of $P$, $\beta_{1}(P)$ is given by $\beta_{1}(P)=b+s-c+1$. If $P$ is tight, each disk-type cluster has degree at least 3 , so each cluster contributes at least $1 / 2$ to $\beta_{1}(P)$. As such, there can be at most 2 clusters in a tight pair of pants and at most 3 strands, and one can enumerate the possibilities.

We can now prove that for each combinatorial type, there are $\lesssim e^{A}$ tight pairs of pants with $A$ triangles. Since there are a finite number of combinatorial types, this will imply the lemma.

By Lemma 4, there is a $c>0$ such that there are at most $e^{c A}$ discs or annuli or three-holed spheres with $A$ triangles. Now to build a tight pair of pants, we have to add strands to the triangulated surfaces. We have to choose the attaching points. There are at most six attaching points, and each attaching point has at most $3 \mathrm{~A}$ choices, and so there are $\leq(3 A)^{6}$ choices of attaching points. Once we have chosen where to attach each strand, the lengths of the strands are determined by the lengths $l_{1}, l_{2}$, and $l_{3}$ of the three boundary circles. Thus the total number of tight pants with fixed boundary lengths, a fixed combinatorial type, and $A$ triangles is bounded by $5 e^{2 c A}(3 A)^{6} \lesssim e^{A}$.

REMARK. In hyperbolic geometry, there is a unique hyperbolic pair of pants for every choice of boundary lengths $l_{1}, l_{2}$, and $l_{3}$. The closest combinatorial analogue of this phenomenon is the fact that tight pairs of pants with no triangles are determined by their boundary lengths. For every triple of lengths, $l_{1}, l_{2}, l_{3} \in \mathbb{Z}$, there is a unique triangle-less pair of pants with the given lengths. If the lengths obey the triangle inequality, the graph looks like $\theta$ and if not the graph looks like a pair of glasses, that is, two circles connected by a line. If we are not careful when we add triangles, the number of pairs of pants explodes: if we add one triangle, we have $\approx l_{1}+l_{2}+l_{3}$ different edges where we can put it, so we get many different pairs of pants. For this reason, we introduced tight pairs of pants; tightness restricts the possible places that a triangle can go. The number of tight pairs of pants with fixed boundary lengths is bounded by $\exp (A)$, with constant independent of the chosen boundary lengths. The $\exp (A)$ factor is fairly harmless, so tight pairs of pants are a good analogue of hyperbolic pairs of pants.

Recall that a combinatorial pants decomposition consisted of a collection of pants and some gluing information. Next we consider combinatorial analogues of length and twist parameters (i.e. Fenchel-Nielsen coordinates) and bound the number of possible ways to glue pants. 
Lemma 8. There is a $C$ such that the number of genus $g$ combinatorial pants decompositions with total pants length $\leq L$ and total area $\leq N$ is bounded by $\leq C g^{g}(L / g)^{6 g} \exp (C N)$.

Proof. In this proof, we will write $f(g, L, N) \lesssim h(g, L, N)$ to mean that there is some $c$ such that $f(g, L, N) \leq c e^{c N} h(g, L, N)$ for all applicable $g, L, N$. Note that we may assume that $g \leq N / 4$, and so $e^{g} \lesssim 1$ as usual.

As with hyperbolic pants decompositions, a genus- $g$ combinatorial pants decomposition has a topological type. By Lemma 1, the number of topological types of genus $g$ is $\approx g^{g}$.

Now we count tight pants decompositions with a fixed topological type. We first have to choose the lengths of the $3 g-3$ boundary curves in the pants decomposition. How many ways can we choose positive integers $l_{1}, \ldots, l_{3 g-3}$ so that $\sum l_{i} \leq L$ ? This number is less than the volume of the set $x_{i} \geq 0, \sum x_{i} \leq L$. The volume of that simplex can be computed by induction on the dimension; it has volume $\frac{1}{(3 g-3) !} L^{3 g-3} \lesssim(L / g)^{3 g}$

For each choice of lengths, we next have to choose how many triangles to put in each pair of pants. Here we have to choose $A_{1}, \ldots, A_{2 g-2} \geq 0$ with $\sum A_{i}=N$. The number of ways to choose the $A_{i}$ is exactly $\left(\begin{array}{c}N+2 g-3 \\ 2 g-3\end{array}\right) \leq 2^{N+2 g-3} \leq 4^{N}$. (Since $g \leq N / 4$.) So the number of ways of choosing $A_{i}$ is $\underset{\sim}{\lesssim} 1$.

Next we have to choose a tight pants structure for each pair of pants with the given area $A_{i}$ and the given boundary lengths. If $c_{0}$ is the constant from Lemma 4 , then the number of ways to do this is at most $\prod_{i} c_{0} \exp \left(c_{0} A_{i}\right) \lesssim 1$.

Now we count the number of possible gluings. Since we already chose the topological type, a gluing is determined by its twist parameters. For each of the $3 g-3$ curves, the twist parameter is an integer in the range $0 \leq t_{i} \leq l_{i}-1$. The number of choices for the twist parameters is

$$
\prod_{j=1}^{3 g-3} l_{j} \leq\left(\frac{L}{3 g-3}\right)^{3 g-3} \lesssim(L / g)^{3 g} .
$$

Multiplying all of these together, we find that the number of possible pants decompositions is

$$
\lesssim g^{g}(L / g)^{3 g}(L / g)^{3 g} \lesssim g^{g}(L / g)^{6 g}
$$

as desired.

In particular, the number of underlying surfaces (up to simplicial isomorphism) is $\lesssim(L / g)^{6 g} g^{g}$. This proves the main estimate.

The total number of combinatorial surfaces in $\operatorname{Comb}_{N}$ is $\approx N^{N / 2}$. If $N$ is sufficiently large and $L=N^{7 / 6-\varepsilon}$, then the number of surfaces in $\mathrm{Comb}_{N}$ with genus $\geq 2$ and total pants length at most $L$ is

$$
\lesssim \sum_{i=2}^{(N+2) / 4} e^{c N}(L / i)^{6 i} i^{i} \lesssim e^{c N} N^{\left(\frac{1}{6}-\varepsilon\right) \cdot \frac{6 N}{4}}(N / 4)^{\frac{N}{4}} \approx N^{N / 2-3 \varepsilon / 2}
$$


The number of surfaces in $\mathrm{Comb}_{N}$ with genus $<2$ is $\lesssim 1$ by Lemma 4 , so for large $N$, most surfaces in $\mathrm{Comb}_{N}$ have no pants decomposition of total length $\leq L$. This finishes the proof of Theorem 2.

\section{References}

[BP] F. BALAChefF, H. PARLier, Bers' constants for punctured spheres and hyperelliptic surfaces, preprint (2009).

[BPS] F. Balacheff, H. Parlier, S. Sabourau, Short loop decompositions of surfaces and the geometry of jacobians, preprint (2010).

[BS] F. Balacheff, S. Sabourau, Diastolic inequalities and isoperimetric inequalities on surfaces, Ann. Sci. École Norm. Sup. 43:4 (2010), 579-605.

[Be1] L. Bers, Spaces of degenerating Riemann surfaces, Discontinuous Groups and Riemann Surfaces (Proc. Conf. Univ. Maryland, College Park, Md., 1973), Ann. of Math. Studies 79, Princeton Univ. Press, Princeton, NJ (1974), 43-55.

[Be2] L. Bers, An inequality for Riemann surfaces, Differential Geometry and Complex Analysis, Springer, Berlin (1985), 87-93.

[Bo] B. Bollobás, The asymptotic number of unlabelled regular graphs, J. London Math. Soc. (2) 26:2 (1982), 201-206.

[BrM] R. Brooks, E. Makover, Random construction of Riemann surfaces, J. Differential Geom. 68:1 (2004), 121-157.

[Bro] W.G. Brown, Enumeration of triangulations of the disk, Proc. London Math. Soc. (3) 14 (1964), 746-768.

[Bu1] P. BusER, Riemannshe flächen und längenspektrum vom trigonometrishen standpunkt, Habilitation Thesis, University of Bonn (1981).

[Bu2] P. Buser, Geometry and Spectra of Compact Riemann Surfaces, Progress in Mathematics 106, Birkhäuser Boston Inc., Boston, MA (1992).

[BuS] P. Buser, P. SARnak, On the period matrix of a Riemann surface of large genus (with appendix by J.H. Conway and N.J.A. Sloane), Invent. Math. 117:1 (1994), $27-56$,

[BuSe] P. Buser, M. SeppäLÄ, Symmetric pants decompositions of Riemann surfaces, Duke Math. J. 67:1 (1992), 39-55.

[G] A. Gamburd, Poisson-Dirichlet distribution for random Belyi surfaces, Ann. Probab. 34:5 (2006), 1827-1848.

[GM] A. Gamburd, E. Makover, On the genus of a random Riemann surface, Complex Manifolds and Hyperbolic Geometry (Guanajuato, 2001), Contemp. Math. 311, Amer. Math. Soc., Providence, RI (2002), 133-140.

[Gr] M. Gromov, Metric Structures for Riemannian and Non-Riemannian Spaces (English ed.), [Based on the 1981 French original, with appendices by M. Katz, P. Pansu and S. Semmes, Translated from the French by S.M. Bates], Modern Birkhäuser Classics, Birkhäuser Boston Inc., Boston, MA (2007),

[Gru] S. Grushevsky, An explicit upper bound for Weil-Petersson volumes of the moduli spaces of punctured Riemann surfaces, Math. Ann. 321:1 (2001), 1-13.

[L] J.-F. LE GALL, The topological structure of scaling limits of large planar maps, Invent. Math. 169:3 (2007), 621-670.

[MM] E. Makover, J. MCGowan, The length of closed geodesics on random Riemann surfaces, Geom. Ded. 151:3 (2011), 207-220. 
[Mi] M. Mirzakhani, Growth of Weil-Petersson volumes and random hyperbolic surfaces of large genus, 2010.

[P] R.C. Penner, Weil-Petersson volumes, J. Differential Geom. 35:3 (1992), 559-608.

[ST] G. Schumacher, S. Trapani, Estimates of Weil-Petersson volumes via effective divisors, Comm. Math. Phys. 222:1 (2001), 1-7.

[W] S.A. Wolpert, Geometry of the Weil-Petersson completion of Teichmüller space, Surveys in Differential Geometry, Vol. VIII (Boston, MA, 2002), Surv. Differ. Geom. VIII, Int. Press, Somerville, MA (2003), 357-393.

Larry Guth, Department of Mathematics, University of Toronto, Bahen Centre, 40 St. George St. Toronto, Ontario, M5S 2E4, Canada lguth@math.utoronto.ca

Hugo Parlier, Department of Mathematics, University of Fribourg, Chemin du Musée 23, CH-1700 Fribourg, Switzerland hugo.parlier@gmail.com

Robert Young, Courant Institute of Mathematical Sciences, 251 Mercer St., New York, NY 10012, USA rjyoung1729@gmail.com

Received: November 2, 2010 Revision: May 18, 2011 Accepted: May 25, 2011 